\title{
The History of Ampersand and its Functions
}

\author{
P. Pandia Rajammal, V. Manoj Prabhakaran, A. G. Vadivelan
}

\begin{abstract}
This paper discusses the importance and contributions one of the most popular characters or symbols of the English Language, which is now not an official alphabet but once played a significant role among all the English Alphabets. Despite its removal from the list of official alphabets, ' $\&$ ' still continues to be used everywhere from literature, formal and informal languages to even computer programming. This explains why ' $\&$ ' is a potential candidate to be brought back to the list of Alphabets.
\end{abstract}

Keywords: Symbols, Alphabets, Literature, Programming, Potential

\section{INTRODUCTION}

The Ampersand which of course had a quite number of symbols for the same word, was indeed used since a long time ago. This special symbol has its origins from the Latin word 'Et' meaning 'And' and which was among some of the symbols that were included as a part of the English Alphabets before the Modern English Society decided to officially remove it from the Alphabets and hence rather becoming just a symbol. This word and was popularly symbolized as ' $\&$ ', that just meant 'And' and was also just said as 'And'. But the fact is that sooner or later, things changed and the symbol ' $\&$ ' was no longer pronounced as 'And' instead it became 'Ampersand'.

The olden English had ' $\&$ ' as its last alphabet back then. This led to the English Alphabets to start from A... B... C... .... X... Y... Z... and \& being its last letter of the list. Also, that was the time when the English Language was popularized with the usage of the word 'Per se', which also, has its roots in Latin word 'Per sei', meaning 'of/by itself'. This hence made the change of how alphabets were said in the order. Mainly, children in schools said the alphabets like... A... B... C...... X... Y... Z... and per se \&! in other words 'and per se and' which simply meant '... and 'and' by itself is an alphabet'! Which in due course got morphed from 'And Per se and' to Ampersand.

This was an official English alphabet and was primarily used as a shorthand for 'and'. But later due to the fact that $\&$ is a standalone symbol and cannot be used as a part of any other word or it cannot be used to form new words, unlike the rest of the alphabets and moreover it was just a substitute for the word 'and', \& was removed from the list of English

Revised Manuscript Received on December 24, 2019.

P. Pandia Rajammal* , English department, Kalasalingam Academy of Research and Education, Krishnankoil, Tamil Nadu, India. pandiarajammal.p@klu.ac.in

Mr. V. Manoj Prabhakaran, IV Year, ICT department, SASTRA Deemed To Be University, Tanjore, Tamil Nadu, India.iammanoj02@gmail.com

A. G. Vadivelan, English department, Kalasalingam Academy of Research and Education, Krishnankoil, Tamil Nadu, India. vadivelanag@gmail.com
Alphabets. Despite being removed from the list of the English Alphabets, ampersand's usage did not end. It was only decided not to place it in the list of Alphabets, not to restrict its usage. In fact, Ampersand was the most popular English character that was not a part of the English Alphabets. The fact is that \& features in a lot of English works and writings even after its removal and till today, it are effectively used as a substitute for the word 'and'.

\section{THE SCRIPT}

Ampersand had almost three basic scripts that were widely used since the ages. The Modern symbol ' $\&$ ' of the ampersand is implemented widely nowadays and is the most common of all the scripts. However, the symbolic representation was similar to an Epsilon was famous back in the days and is still used in some places. The Modern representation ' $\&$ ' looks like a mutant of its origin word 'et', where the two letters, by usage has joined to form a single symbol.

\section{USAGE}

\section{A. Usage across Years:}

It is of no doubt that ' $\&$ ' is widely used as a potential substitute or a shorthand for the word 'and'. With its first use in 1795 , it soon became a popular representation and an integral part of the Language. By 1837, this symbol had already entered into common English usage. In M. B. Moore's 1863 book, "The Dixie Primer, for the Little Folks", the symbol ' $\&$ ' was already included as the 27 th letter of the English Alphabet. It was also a key point to note that some of the characters such as Thorn, Eth, Wynn, etc., were removed. Later, Ampersand was removed from the official list of alphabets but its widespread usage did not cease. It continued to be in common usage due to its popularity.

Now, Ampersand's use is quite constrained. The English language currently does not allow \& as an exact substitute for 'and' always. In some cases, 'and' is lot preferred than ' $\&$ '. These rules are implicitly followed in usage. Apart from the linguistic usage of ' $\&$ ', it is widely used in a lot of computer programming languages like $\mathrm{C}, \mathrm{C}++$, Java, etc.

General Rules Involved in the Usage:

- First of all, the ampersand is generally not allowed to be used as an absolute substitute for the word 'and' in formal language but it is permitted to use it as a substitute in case of informal language.

- Logos, titles, shorthand expressions may contain ' $\&$ ' in its formal usage too.

- While writing addresses it is permitted to use ' $\&$ ' in-between them. 
- The ampersand can be used inside citations and inside the parenthesis, where there is not much space available.

\section{B. Usage and Importance in Modern World:}

Ampersand, as discussed above, is being used in modern times, despite its removal from the set of English alphabets. With all implicit rules employed in using the symbol, it still proves to be an effective part of the language. In the world of internet, there is always a need for conveying information as short as possible. We can surely tell that ' $\&$ ' is very unique from all other symbols of the English language because of its shorthand purpose, which no other symbol has.

This has been an attractive advantage for a lot of companies and industries in their respective names. Hence it looks better to add' $\&$ ' in their names than using the word 'and'. The same is true when it comes to the design of logos. Modern-day logos prefer shorthand notations than real and large names. The ' $\&$ ' symbol proves its advantage here also. There are numerous institutions, companies and organizations that have ' $\&$ ' in their respective logos. Modern newspapers, articles, etc, also need shorthand, thereby promoting the advantage of ' $\&$ '.

\section{Usage of ' $\&$ ' in Computer Programming Languages:}

When it comes to the usage of the symbol ' $\&$ ', Computer programming languages deserve a special mention. The symbol ' $\&$ ' was initially used as a symbolic notation of the 'Boolean Operator - AND' which was a head start for the symbol to be directly included in almost all of the computer programming languages - ranging from simple to complex languages. Even today almost every important computer language from $\mathrm{C}, \mathrm{C}++$ to Python, use ' $\&$ ', which has anchored its role in those languages. Apart from programming languages, Computer Operating Systems languages like UNIX, LINUX, etc., also implicitly use ' $\&$ ' because of the important role of ampersand played in all those instances.

\section{Usage of ' $\boldsymbol{~} \boldsymbol{\text { ' }}$ in the Mathematical world:}

It is also an important fact to mention that the special symbol is not just used in programming only. It is also deployed in the domain of logic and mathematics. As mentioned above, the symbol is used as a very important shorthand for the mathematical operation 'AND'. This has made it so indispensable in the domain of logic and inference in the field of mathematics. The same symbol is also used in the set theory domains of mathematics. This hence proves that the role of ' $\&$ ' is very important and has already played a great role in mathematics and even science too, remaining as an essential one in the future too.

\section{ROLE IN FUTURE}

As discussed above, due to the important role played by ' $\&$ ' in a variety of applications and fields, it's almost impossible to find a substitute for the symbol in those cases hence it will be highly essential even in the future. It is highly evident that the usage of ampersand will never diminish in the future.
With the above fact said, it is clear that it would really be a good idea to make ampersand an official English letter again.

\section{A Potential Candidate for the 27th letter:}

It is a well-known fact that ' $\&$ ' was the 27 th letter of the list of English alphabets, which was later removed due to the reasons which were discussed above. Despite being removed as an official alphabet, ' $\&$ ' unlike the rest of the alphabets that were removed, remains in the common usage. In fact, the usage of the symbol ' $\&$ ' has quite increased in the modern world thanks to the promotions that were received from the computer programming languages and other modern requirements.

It is an important fact that ' $\&$ ' still remains in the official list of English Characters, despite its exclusion from the list of official alphabets. Hence if the English Literary Society recognizes the importance, potential and usage of this symbol and changes the criteria for alphabets, it is sure that Ampersand is the character with the highest priority to be included back into the English Alphabets.

\section{CONCLUSION}

The above paper has discussed the history, usage, importance and the potentiality for the letter ' $\&$ ' and has even explained why this special character, which was once included in the list of alphabets, could be brought into the list yet again, becoming the 27 th letter without any much surprise.

\section{REFERENCES}

1. "The ampersand". word-detective. Archived from the original on 8 May 2008.

2. Robert Hartwell Fiske's Dictionary of Unendurable English: A Compendium of Mistakes in Grammar, Usage, and Spelling with Commentary on Lexicographers.

3. https://medium.com/black-lion-banner/the-history-of-the-ampersand.

4. https://www.dictionary.com/e/ampersand/

\section{AUTHORS PROFILE}

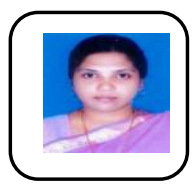

Dr. P. Pandia Rajammal, PhD., graduated at Madurai Kamaraj University, Tamil Nadu, India, presented papers at National and International Conference, published papers in Journal, Organied seminars and Guest Lectures, Guided PG projects and MPhil dissertations, working as an Assistant Education, Tamil Nadu, India. Professor at Kalasalingam Academy of Research and

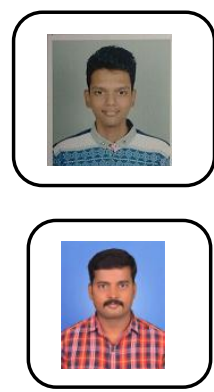

V. Manoj Prabhakaran, IV Year ICT Department, SASTRA Deemed To Be University, Published a paper on Survey of Journal of Advanced Research in Dynamical \& Control Systems, Participated in Slope protection systems (Smart India Hackathon - Final Round.

Dr. A. G. Vadivelan, PhD., graduated at Anna University, Tamil Nadu, India, presented and published papers at National and International Conference. Published papers at International Journals. Guided UG, PG Projects and MPhil Dissertation. 